\title{
Two new species of Aivalykus (Hymenoptera: Braconidae: Doryctinae) from China and Indonesia, with a key to species
}

\author{
Sergey A. BELOKOBYLSKIJ ${ }^{1}$ and XueXIN CHEN ${ }^{2}$ \\ ${ }^{1}$ Zoological Institute, Russian Academy of Sciences, Universitetskaya nab., 1, St.Petersburg 199034, Russia; e-mail: \\ hymenopt@zin.ru \\ ${ }^{2}$ Department of Plant Protection and Institute of Applied Entomology, College of Agriculture and Biotechnology, Zhejiang \\ University, Huajiachi Campus, Hangzhou 310029, China
}

Key words. Taxonomy, Hymenoptera, Braconidae, Doryctinae, Aivalykus, new species, new synonymy, new combination, China

\begin{abstract}
The species and tribal position of the genus Aivalykus Nixon are discussed. Two new species of Aivalykus from China and Indonesia are described: $A$. nitidus sp. n. and $A$. bouceki sp. n. The genus Ecphyloides Marsh, 1993 is considered a junior synonym of Aivalykus Nixon, 1938. A key to the species of Aivalykus is given.
\end{abstract}

\section{INTRODUCTION}

The genus Aivalykus was described by G.E.J. Nixon (1938) for two species from India - A . eclectes Nixon, 1938 (type species of the genus, which was also recorded in the Philippines) and $A$. sperches Nixon, 1938. Later additional species of this genus were described from Madagascar (A. niger Granger, 1949; but the status of this species must be verified) and the Southern Nearctic ( $A$. nearcticus Marsh, 1965). Recently P. Marsh (1993) described a new genus from Brazil, Ecphyloides Marsh, 1993, with type species E. flavus Marsh, 1993. The main difference between Ecphyloides and Aivalykus is the number of the antennal segments (Marsh, 1993, 1997) (Ecphyloides has 14-segmented antennae). However, the known variation in the number of antennal segments in species of the most closely related genus Ecphylus Förster, 1862 range from 11 (E. schwarzii (Ashmead, $1900)$ ) to 26 (E. hicoriae Rohwer, 1919), and within species (for example) E. hypothenemi Ashmead, 1896 - from 13 to 16 , or E. schwarzii - from 11 to 14 (Marsh, 1965). Additionally P. Marsh (1965) in the description of Aivalykus nearcticus (p. 677) noted that the number of antennal segments varied from 14 to 19 . Because of this variation, and the study of the holotype of $E$. flavus in the Canadian National Collection (Ottawa) (female, "Brasilien, Rio Caraguala, $21^{\circ} 48^{\prime} \mathrm{B} .52^{\circ} 27^{\prime} \mathrm{L}, 400 \mathrm{~m}$, III-1953, Fritz Plaumann", "Holotype Ecphyloides flavus Marsh") by the first author, we propose to synonymize Ecphyloides Marsh, 1993 with Aivalykus Nixon, 1938 (syn. n.). In addition, two new species of Aivalykus from China and Indonesia are described below.

The genus Aivalykus was placed originally in the tribe Hecabolini (Nixon, 1938; Marsh, 1965; Shenefelt \& Marsh, 1976). But a recent reclassification of the infrageneric taxa of the subfamily Doryctinae has indicated the apomorphic characters of the tribe Ecphylini Hellén, 1957, separating it from the tribe Hecabolini Förster, 1862 (Belokobylskij, 1992). On the basis of these charac- ters, Aivalykus is considered a member of the tribe Ecphylini (together with Ecphylus Förster, 1862 and Bohartiellus Marsh, 1983), because they both lack a nervellus and recurrent vein in the hind wing, and the brachial cell of fore wing is closed apically and the parallel vein has an interstitial position. The same tribe for Aivalykus was also proposed by J. Papp (1993).

Some species of Aivalykus are known to be parasites of larvae of Scolytidae (genera Sphaerotrypes Blandford, 1894, Phthorophloeus Rey, 1883, Phloeosinus Chapuis, 1873), which are the main hosts of all members of the tribe Ecphylini. It is very possible that, on the basis of the small size of the specimens examined in this study, the hosts of the new species might also be scolytid larvae.

The terminology for wing venation follows that of Belokobylskij \& Tobias (1998). The following abbreviations are used: POL - postocellar line; OOL - ocularocellar line; Od - maximum diameter of lateral ocellus; BMNH - The Natural History Museum (London, England); RMNH - Nationaal Natuurhistorisch Museum (Leiden, Netherlands); ZISP - Zoological Institute, Russian Academy of Sciences (St. Petersburg, Russia); ZUH - Zhejiang University (Hangzhou, China).

\section{Aivalykus Nixon, 1938}

Aivalykus Nixon, 1938: 152. Type species Aivalykus eclectes Nixon, 1938 (original designation).

Ecphyloides Marsh, 1993: 14. Type species Ecphyloides flavus Marsh, 1993, syn. n.

\section{Aivalykus nitidus sp. $n$.}

(Figs 1-13)

Type material. Holotype: female, China, "Henan, Mt. Jigong, 1997.VII.12, Chen Xuexin, N 974980" (ZUH). Paratypes. 4 females, 1 male, "Java, M., Telawa, VIII-31. J. 53, L.G.E. Kalshoven" (RMNH, ZISP).

Description. Female. Body length $2.0-2.2 \mathrm{~mm}$; fore wing length $1.8-2.0 \mathrm{~mm}$. Head width $1.5-1.6$ times its median length. Temple roundly narrowed behind eye; 


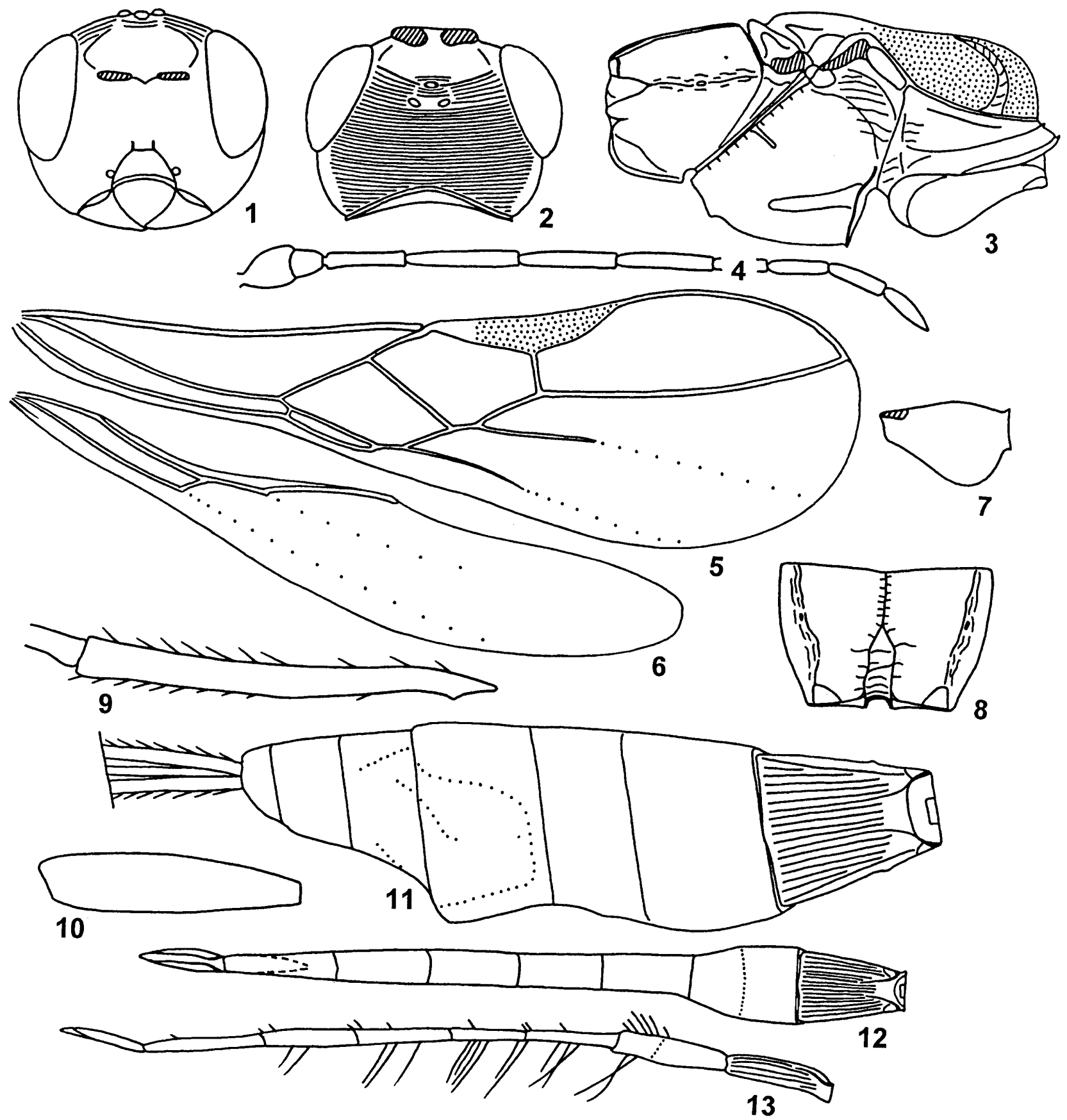

Figs 1-13. Aivalykus nitidus sp. n. 1-11 - female; 12, 13 - male. 1 - head, frontal view; 2 - head, dorsal view; 3 - metasoma, lateral view; 4 - basal and apical segments of antenna; 5 - fore wing; 6 - hind wing; 7 - hind coxa; 8 - propodeum; 9 - hind tibia; 10 - hind femur; 11, 12 - metasoma, dorsal view; 13 - metasoma, lateral view.

transverse diameter of eye 1.8-2.1 times length of temple. Ocelli small, in triangle with base 1.1-1.3 times its sides. POL 1.0-1.25 times Od, 0.3-0.35 times OOL. Eye glabrous, 1.1-1.15 times as high as broad. Malar space height 0.4 times height of eye, $0.85-1.0$ times basal width of mandible. Face width almost equal to height of eye and 1.2-1.4 times height of face and clypeus combined. Malar suture absent. Clypeus with narrow lower flange. Clypeal suture shallow, but distinct. Hypoclypeal depression round, its width $0.7-0.8$ times distance from edge of depression to eye. Occipital carina reduced below hence not fused with the hypostomal carina.
Antennae filiform, 21-segmented, 1.2 times as long as body. Scapus 1.2-1.3 times as long as its maximum width. First flagellar segment not flattened, weakly curved, 4.5-4.8 times as long as its apical width, 0.7 times as long as second segment. Penultimate segment 4.3 times as long as wide, 0.7 times as long as first flagellar segment, as long as apical segment, which is pointed apically.

Mesosoma. Length 1.8-1.9 times its height. Neck of promesosoma very short. Pronotal carina distinct. Mesoscutum highly and almost perpendicularly raised above pronotum. Notauli deep in anterior half, very 
shallow or almost absent in posterior half, sparsely crenulate. Prescutellar depression deep, with distinct median carina, very finely rugulose, 0.3 times as long as scutellum. Subalar depression shallow, wide, very finely rugulose. Sternauli distinct, smooth, connected with prepectal carina anteriorly, running along approximately half of lower part of mesopleura. Propodeum without lateral tubercles.

Wings. Fore wing $3.5-3.8$ times as long as its maximum width. Radial vein arising from or behind middle of pterostigma. Radial cell not shortened. First radial abscissa almost perpendicular to pterostigma, 0.8-1.0 times as long as maximum width of pterostigma, 0.6-0.7 times as long as first radiomedial vein. Second radial abscissa weakly curved, 6.8-7.8 times as long as first abscissa, about 4.5 times as long as first radiomedial vein. Discoidal cell 2.1-2.3 times as long as wide. Recurrent vein antefurcal, 1.6-2.0 times as long as second abscissa of medial vein. Brachial cell narrow, gently closing apically distinctly before the recurrent vein. Distance from nervulus to basal vein $1.0-1.5$ times nervulus length. In hind wing medial cell closed anteriorly.

Legs. Hind femur 3.8-4.4 times as long as wide. Hind coxa without basoventral tooth. Hind tarsus 0.9 times as long as hind tibia. Basitarsus $0.7-0.75$ times as long as second-fifth segments combined. Second tarsal segment $0.4-0.45$ times as long as basitarsus, about 1.3 times as long as fifth segment (without pretarsus).

Metasoma 0.85-1.1 times as long as head and mesosoma combined. First tergite without spiracular tubercles, spiracles situated on basal $1 / 3$, weakly and linearly widened from base to apex. Maximum width of first tergite 1.7 times its width at level of dorsope; length 1.1-1.2 times its apical width. Second tergite without depressions, length of tergite 0.8 times its basal width, 1.4 times length of third tergite. Second suture shallow and straight. Ovipositor sheath $0.6-0.8$ times as long as body, 1.2-1.7 times as long as metasoma, 1.7-2.2 times as long as mesosoma, $0.7-0.8$ times as long as fore wing.

Sculpture and pubescence. Vertex very densely and finely aciculate; frons almost entirely and temple smooth; face very finely and sparsely rugulose. Sides of pronotum almost entirely smooth. Mesoscutum granulate, finely and narrowly striate medioposteriorly. Scutellum almost smooth. Mesopleura and metapleura smooth. Propodeum smooth, with fine rugulosity along carinae, with median carina in basal half and with distinctly marginate, long, narrow and striate areola in apical half. Hind legs smooth. First metasomal tergite with distinct and complete dorsal carinae, densely striate, finely rugulose medially between dorsal carinae. Rest of tergites smooth. Hind tibia with rather sparse and semierect hairs dorsally, length of these hairs 0.7-0.8 times maximum width of hind tibia.

Colour. Head and anterior half of mesosoma reddish yellow, posterior half of mesosoma and entire metasoma reddish brown, sometimes darker or lighter. Two or three basal segments of antenna yellow, rest of segments reddish brown to dark reddish brown, 3 apical segments white. Palpi yellow. Legs entirely pale yellow or yellow.
Ovipositor sheath black. Wings hyaline. Pterostigma brown, paler in basal third.

Male. Body length $3.8 \mathrm{~mm}$; fore wing length $1.9 \mathrm{~mm}$. Recurrent vein of fore wing as long as second medial abscissa. Metasoma very long, strongly narrowed behind third tergite, then almost parallel-sided and depressed; 2.5 times as long as mesosoma and head combined. Length of first tergite 1.4 times its apical width. Metasoma behind third tergite ventrally with several sparse long hairs; dorsal hairs along border of tergites significantly shorter. Otherwise similar to female.

Discussion. This new species is similar to A. sperches from India, but differs in the shorter ovipositor, smooth second metasomal tergite, the 3 apical segments of antenna being white, the first flagellar segment being distinctly shorter than the second segment, and presence of sternauli.

Etymology. From Latin "nitidus" (shining), because most of the body of this species is shiny.

\section{Aivalykus bouceki sp. n.}

\section{(Figs 14-24)}

Type material. Holotype: female, "China, Hainan I., Tien Fong Mts, V.[19]83, Bouček" (BMNH).

Description. Female. Body length $3.0 \mathrm{~mm}$; fore wing length $2.5 \mathrm{~mm}$. Head width 1.4 times its median length. Temple distinctly roundly narrowed behind eye; transverse diameter of eye 1.6 times length of temple. Ocelli small, in triangle with base 1.3 times its sides. POL almost equal to Od, 0.4 times OOL. Eye glabrous, 1.4 times as high as broad. Malar space height 0.4 times height of eye, 0.8 times basal width of mandible. Face width 0.9 times height of eye and 1.1 times height of face and clypeus combined. Malar suture absent. Clypeus with narrow lower flange. Clypeal suture distinct. Hypoclypeal depression almost round, its width 0.7 times distance from edge of depression to eye. Occipital carina reduced below hence not fused with the hypostomal carina. Palpi long, length of maxillary palpi about 1.7 times head height.

Antennae filiform, 27-segmented, 1.3 times as long as body. Scapus about as long as its maximum width. First flagellar segment not flattened, weakly curved, almost 5 times as long as its apical width, 0.6 times as long as second segment. Penultimate segment 4.5 times as long as wide, 0.6 times as long as first flagellar segment, as long as apical segment, which is pointed apically.

Mesosoma. Length almost twice its height. Neck of promesosoma very short. Pronotal carina distinct. Mesoscutum highly and almost perpendicularly raised above pronotum. Notauli deep in anterior half, shallow in posterior half, sparsely crenulate. Prescutellar depression rather deep, with distinct median carina, very finely rugulose, 0.3 times as long as scutellum. Subalar depression shallow, wide, partly finely rugulose. Sternauli deep, almost smooth, connected with prepectal carina anteriorly, running along approximately $2 / 3$ of lower part of mesopleura. Propodeum without lateral tubercles. 

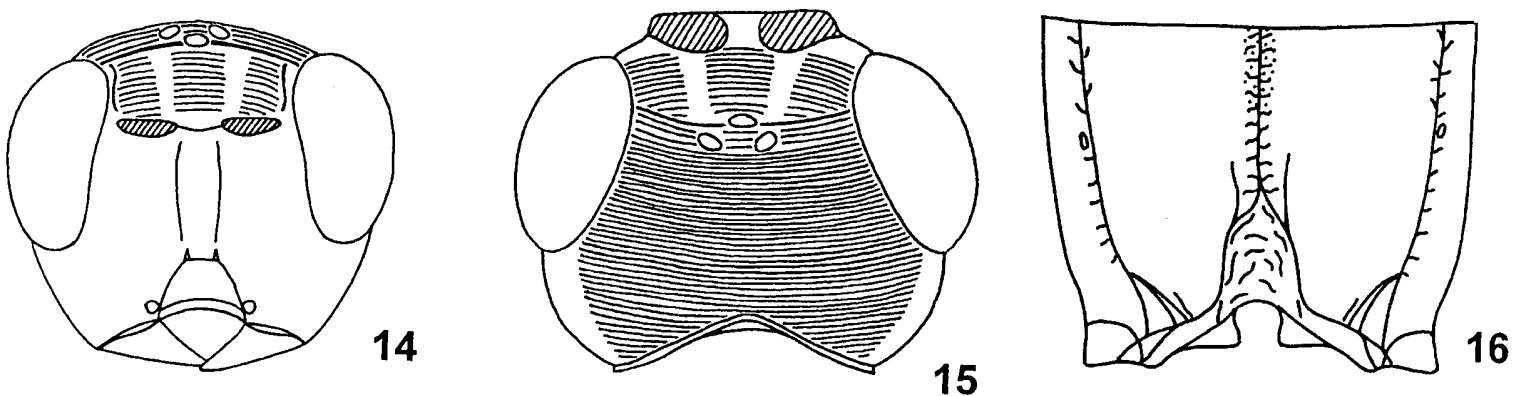

15

16
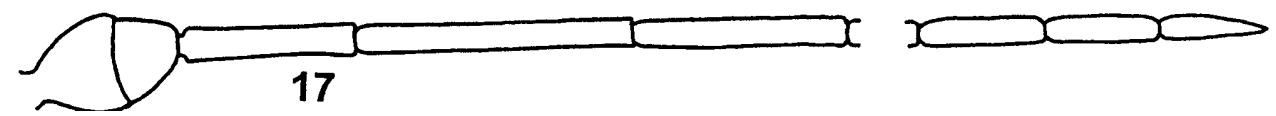

17
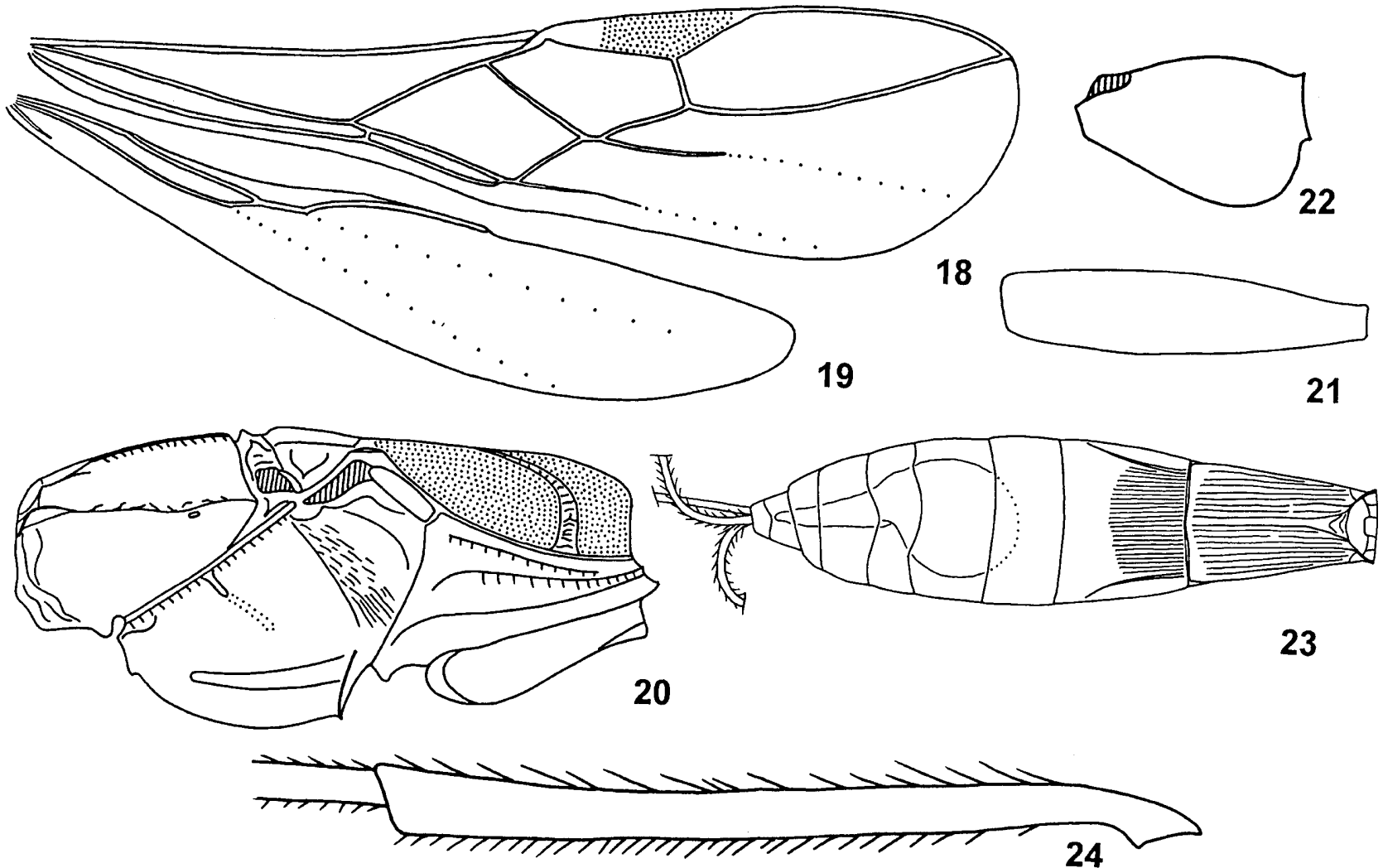

Figs 14-24. Aivalykus bouceki sp. n. 14 - head, frontal view; 15 - head, dorsal view; 16 - propodeum; 17 - basal and apical segments of antenna; 18 - fore wing; 19 - hind wing; 20 - mesosoma, lateral view; 21 - hind femur; 22 - hind coxa; 23 - metasoma, dorsal view; 24 - hind tibia.

Wings. Fore wing 3.8 times as long as its maximum width. Radial vein arising behind middle of pterostigma. Radial cell not shortened. First radial abscissa almost perpendicular to pterostigma, almost equal to maximum width of pterostigma, 0.6 times as long as first radiomedial vein. Second radial abscissa weakly curved, 6.5 times as long as first abscissa, 4 times as long as first radiomedial vein. Discoidal cell almost twice as long as wide. Recurrent vein antefurcal, 4.2 times as long as second abscissa of medial vein. Brachial cell narrow, gently closing apically weakly before the recurrent vein. Distance from nervulus to basal vein almost twice length of nervulus. In hind wing medial cell closed anteriorly.
Legs. Hind femur 4.2 times as long as wide. Hind coxa without basoventral tooth. Hind tarsus 0.9 times as long as hind tibia. Basitarsus 0.65 times as long as second-fifth segments combined. Second tarsal segment 0.6 times as long as basitarsus, twice as long as fifth segment (without pretarsus).

Metasoma 1.1 times as long as head and mesosoma combined. First tergite without spiracular tubercles, spiracles situated on basal third, distinctly and almost linearly widened from base to apex. Maximum width of first tergite 1.8 times its minimum width; length 1.4 times its apical width. Second tergite with fine lateral furrows, which are parallel to tergite margins; length of tergite almost equal to its basal width, 1.7 times length of third 
tergite. Second suture shallow and regularly curved. Ovipositor sheath slightly longer than body, almost twice as long as metasoma, 3 times as long as mesosoma, 1.2 times as long as fore wing.

Sculpture and pubescence. Vertex very densely and distinctly aciculate; frons finely aciculate, partly smooth; face finely rugulose submedially and mostly smooth. Sides of pronotum mostly smooth, finely rugulose-striate anteriorly and posteriorly. Mesoscutum densely and finely granulate, with 2 subparallel striae medioposteriorly. Scutellum smooth. Mesopleura and metapleura smooth. Propodeum smooth, rugulose along carinae, with median carina on basal $2 / 3$ and with finely marginate, small subtriangular areola. Hind legs smooth. First metasomal tergite with distinct, complete and strongly convergent dorsal carinae; first tergite entirely and basal half of second distinctly striate. Rest of tergites smooth. Hind tibia with short, rather sparse and semierect hairs dorsally, length of these hairs $0.6-0.8$ times maximum width of hind tibia.

Colour. Head brownish yellow. Mesosoma and metasoma light reddish yellow, second tergite laterally and posteriorly, and apex of metasoma, yellow. Antenna reddish brown, 2 basal segments light reddish brown. Palpi yellow. Legs entirely yellow. Ovipositor sheath brown. Wings subhyaline. Pterostigma brown, paler in basal third and apically.

Male unknown.

Discussion. This species is similar to A. eclectes from India, but differs in the first flagellar segment being shorter and weakly curved, the antefurcal recurrent vein, the shorter second segment of the hind tarsus and first metasomal tergite, only the basal half of the second tergite being striate, and the ovipositor sheath being slender. How this species differs from $A$. nitidus sp. $\mathrm{n}$. is presented in the following key.

1. Antenna 21-segmented. Scapus $1.2-1.3$ times as long as maximum width. Brachial cell closed apically distinctly before recurrent vein. Second tarsal segment 1.3 times as long as fifth segment (without pretarsus). Length of first tergite 1.1-1.2 times apical width. Second tergite entirely smooth. Ovipositor shorter, its sheath $0.7-0.8$ times as long as fore wing. Body length $2.0-2.2 \mathrm{~mm} \ldots$... A nitidus sp. $\mathrm{n}$.

- Antenna 27-segmented. Scapus about as long as maximum width. Brachial cell closed apically weakly before recurrent vein. Second tarsal segment twice as long as fifth segment (without pretarsus). Length of first tergite 1.5 times apical width. Second tergite striate in basal half. Ovipositor longer, its sheath 1.2 times as long as fore wing. Body length 3.0 $\mathrm{mm} \ldots \ldots \ldots \ldots \ldots \ldots \ldots$. . bouceki sp. $\mathrm{n}$

Etymology. Named after Z. Bouček, a well-known hymenopterist and collector of the holotype.

\section{Key to the World species of the genus Aivalykus Nixon}

1. Recurrent vein postfurcal or interstitial to first radiomedial vein ........................... 2

- Recurrent vein distinctly antefurcal to first radiomedial vein
2. Head transverse. Vertex transversally striate. Antennae 30 35 -segmented. First metasomal tergite twice as long as apical width. Second and third metasomal tergites mostly longitudinally striate. Second suture absent. Ovipositor sheath as long as body. Body length 4.5-7.0 mm - India, Philippines, Indonesia (Borneo) ….A. eclectes Nixon

- Head subcubical. Vertex coriaceous. Antennae 25-segmented. First metasomal tergite about 1.5 times as long as apical width. Second and third metasomal tergites entirely smooth. Second suture distinct. Ovipositor sheath as long as metasoma. Body length $2.0 \mathrm{~mm}$ - Madagascar ........... A. niger Granger

3. Second metasomal tergite of female entirely smooth. Antennae 14-21-segmented. - Brachial cell closed distinctly before recurrent vein . . . . . . . . . . . . . . . 4

- Second metasomal tergite of female striate at least on basal 1/3. Antennae 25-28-segmented . . . . . . . . 6

4. Vertex smooth. Body length $1.0-1.5 \mathrm{~mm}$. - Brazil ..... ................... A. flavus (Marsh), comb. n.

- Vertex distinctly transversally striate $\ldots \ldots \ldots \ldots \ldots 5$

5. Mesoscutum shallowly punctate. Prescutellar depression with 7 cross carinae. Nervulus interstitial. Length of first tergite less than its apical width. Ovipositor sheath about half as long as metasoma. Body length $1.5-2.5 \mathrm{~mm}$. - USA ......................... A. nearcticus Marsh

- Mesoscutum densely granulate. Prescutellar depression with 1 median cross carina. Nervulus postfurcal. Length of first tergite 1.1-1.2 times its apical width. Ovipositor sheath 1.2-1.7 times as long as metasoma. Body length 2.0-3.8 $\mathrm{mm}$. - China, Indonesia (Java) ....... A. nitidus sp. n.

6. Second metasomal tergite without sublateral longitudinal furrows. Sternauli absent. Brachial cell closed apically on level of recurrent vein. - First antennal segment 0.75 times as long as second segment. Body length $4.0-4.5 \mathrm{~mm}$. - India .......................... A. sperches Nixon

- Second metasomal tergite with distinct sublateral longitudinal furrows. Sternauli present. Brachial cell closed apically before level of recurrent vein $\ldots \ldots \ldots \ldots \ldots \ldots .7$

7. Transverse diameter of eye about twice length of temple. First flagellar segment 0.8 times as long as second. Radial vein arising almost from middle of pterostigma. Brachial cell apically closed significantly before recurrent vein. Length of first metasomal tergite almost equal to or slightly longer than its apical width. Hind tibia with dense dorsal hairs. Body length 2.8-3.8 (female) and 6.0-7.0 (male) mm. - Ghana ............... A. endroedyyoungai Papp

- Transverse diameter of eye 1.6 times length of temple. First flagellar segment 0.6 times as long as second. Radial vein arising behind middle of pterostigma. Brachial cell apically closed weakly before recurrent vein. Length of first metasomal tergite 1.5 times its apical width. Hind tibia with sparse dorsal hairs. Body length $3.0 \mathrm{~mm}$. - China

$$
\ldots \ldots \ldots \ldots \ldots \ldots \ldots \text {. . bouceki sp. } \mathrm{n} \text {. }
$$

ACKNOWLEDGEMENTS. We wish to express our sincere thanks to Dr. D. Quicke and Dr. M. Fitton (London) and Dr. J. Papp (Budapest) for allowing us to study material from the collections of the Natural History Museum and the Hungarian Natural History Museum.

\section{REFERENCES}

BelokobylskiJ S.A. 1992: On the classification and phylogeny of the braconid wasps subfamilies Doryctinae and Exothecinae (Hymenoptera: Braconidae). Part 1. On the classification. 1. Entomol. Obozr. 71: 900-928 (In Russian). 
BelokobylskiJ S.A. \& Tobias V.I. 1998: Introduction. In Lehr P.A. (ed.): Keys to the Insects of the Russian Far East 4(3). Dal'nauka, Vladivostok, pp. 8-26 (in Russian).

Granger C. 1949: Braconides de Madagascar. Memoires de l'Institut Scientifique de Madagascar. Seria A. Biol. Anim. 2: $1-428$.

MARSH P.M. 1965: The Nearctic Doryctinae. I. A review of the subfamily with a taxonomic revision of the tribe Hecabolini (Hymenoptera: Braconidae). Ann. Entomol. Soc. Am. 58: 668-699.

Marsh P.M. 1993: Descriptions of new Western Hemisphere genera of the subfamily Doryctinae (Hymenoptera: Braconidae). Contr. Am. Entomol. Inst. 28: 1-58.
Marsh P.M. 1997: Subfamily Doryctinae. In Wharton R.A., Marsh P.M. \& Sharkey M.J. (eds): Manual of the New World Genera of the Family Braconidae (Hymenoptera) 1. Washington, The International Society of Hymenopterists, pp. 207-233.

Nixon G.E.J. 1938: A new genus of Hecabolinae and a note on the genus Telebolus Marshall (Hym.: Braconidae). Proc. Roy. Entomol. Soc. London, B Taxonomy 7: 152-156.

PAPP J. 1993: Aivalykus endroedyyoungai sp. nov. from Ghana. Entomofauna 14: 313-320.

Shenefelt R.D. \& Marsh P.M. 1976: Hymenopterorum Catalogus. Pars 13. Braconidae 9. Doryctinae. Dr. W. Junk, 's-Gravenhage, pp. 1263-1424.

Received June 25, 2001; revised October 5, 2001; accepted December 6, 2001 